\title{
Mechanically-Tunable Quantum Interference in Ferrocene-Based Single-Molecule Junctions
}

María Camarasa-Gómez, Daniel Hernangómez-Pérez, Michael S. Inkpen, Giacomo Lovat, E-Dean Fung, Xavier Roy, Latha Venkataraman, Ferdinand Evers

Submitted date: 06/05/2020 - Posted date: 07/05/2020

Licence: CC BY-NC-ND 4.0

Citation information: Camarasa-Gómez, María; Hernangómez-Pérez, Daniel; Inkpen, Michael S.; Lovat, Giacomo; Fung, E-Dean; Roy, Xavier; et al. (2020): Mechanically-Tunable Quantum Interference in Ferrocene-Based Single-Molecule Junctions. ChemRxiv. Preprint.

https://doi.org/10.26434/chemrxiv.12252059.v1

Ferrocenes are ubiquitous organometallic building blocks that comprise a Fe atom sandwiched between two cyclopentadienyl $(\mathrm{Cp})$ rings that rotate freely at room temperature. Of widespread interest in fundamental studies and real-world applications, they have also attracted some interest as functional elements of molecular-scale devices. Here we investigate the impact of the configurational degrees of freedom of a ferrocene derivative on its single-molecule junction conductance. Measurements indicate that the conductance of the ferrocene derivative, which is suppressed by two orders of magnitude as compared to a fully conjugated analog, can be modulated by altering the junction configuration. Ab initio transport calculations show that the low conductance is a consequence of destructive quantum interference effects that arise from the hybridization of metal-based $\mathrm{d}$-orbitals and the ligand-based $\pi$-system. By rotating the $\mathrm{Cp}$ rings, the hybridization, and thus the quantum interference, can be mechanically controlled, resulting in a conductance modulation that is seen experimentally.

File list (2)

ferrocene_main.pdf (0.97 MiB)

view on ChemRxiv - download file

ferrocene_SI.pdf (8.17 MiB)

view on ChemRxiv • download file 


\title{
Mechanically-tunable Quantum Interference in Ferrocene-based Single-Molecule
} Junctions

María Camarasa-Gómez ${ }^{\ddagger 1}$, Daniel Hernangómez-Pérez ${ }^{\ddagger 1,2}$, Michael S. Inkpen ${ }^{3 *}$, Giacomo Lovat $^{3}$, E-Dean Fung ${ }^{3}$, Xavier Roy ${ }^{4}$, Latha Venkataraman ${ }^{3,4 *}$, Ferdinand Evers ${ }^{1 *}$

${ }^{1}$ Institute of Theoretical Physics, University of Regensburg, 93040 Regensburg, Germany

${ }^{2}$ Department of Materials and Interfaces, Weizmann Institute of Science, Rehovot 761001, Israel ${ }^{3}$ Department of Applied Physics and Applied Mathematics, Columbia University, New York, New York 10027, United States

${ }^{4}$ Department of Chemistry, Columbia University, New York, New York 10027, United States $¥$ equal contribution

Email: inkpen@usc.edu; 1v2117@columbia.edu; ferdinand.evers@ur.de

\begin{abstract}
Ferrocenes are ubiquitous organometallic building blocks that comprise a $\mathrm{Fe}$ atom sandwiched between two cyclopentadienyl $(\mathrm{Cp})$ rings that rotate freely at room temperature. Of widespread interest in fundamental studies and real-world applications, they have also attracted some interest as functional elements of molecular-scale devices. Here we investigate the impact of the configurational degrees of freedom of a ferrocene derivative on its single-molecule junction conductance. Measurements indicate that the conductance of the ferrocene derivative, which is suppressed by two orders of magnitude as compared to a fully conjugated analog, can be modulated by altering the junction configuration. A $b$ initio transport calculations show that the low conductance is a consequence of destructive quantum interference effects that arise from the hybridization of metal-based d-orbitals and the ligand-based $\pi$-system. By rotating the Cp rings, the hybridization, and thus the quantum interference, can be mechanically controlled, resulting in a conductance modulation that is seen experimentally.
\end{abstract}

Keywords: single-molecule junctions, destructive quantum interference, $\mathrm{d}-\boldsymbol{\pi}$ hybridization, ferrocene 
Metallocenes are archetypical organometallic compounds comprising a single metal atom sandwiched between two cyclopentadienyl (Cp) rings. Since the discovery of ferrocene in the $1950 \mathrm{~s}^{1,2}($ metal atom $=\mathrm{Fe})$, a variety of metallocenes and related sandwich organometallic structures have been synthesized and investigated. ${ }^{3,4}$ One of the key features of ferrocene is that the barrier for the rotation of $\mathrm{Cp}$ rings is low $(\sim 0.05 \mathrm{eV}){ }^{5,6}$ The easy rotation provides flexibility for adsorption on metal surfaces, while also enabling ferrocene-based molecular wires to potentially adopt a range of geometries ${ }^{7}$. Remarkably, the impact of such conformational degrees on electron transport across ferrocene based wires has not been demonstrated previously ${ }^{8}$ despite their use as key elements of rectifiers ${ }^{9,10}$, switches ${ }^{11}$ or spin filters ${ }^{12,13}$. The consequences of the rotational flexibility of ferrocene on transport characteristics have only been computationally addressed recently ${ }^{14-21}$. Variations in the conductance by orders of magnitude have been predicted, which have been attributed to the sensitivity of the molecular geometry to the binding configuration $^{16}$ and/or to differences in Fermi level alignment of several molecular conformations in the junction. ${ }^{15}$

Here we undertake an experimental and theoretical study of coherent charge transport through single-branched ferrocene-based molecular junctions attached to gold electrodes. Using a series of model compounds, we find that single-molecule junctions comprising a ferrocene moiety present complex transport characteristics that can be attributed to quantum interference related to the metal-based d-orbitals. Our experiments show ferrocene-derivatives have a lower conductance than a conjugated organic analog of similar length while enabling a larger change of conductance upon mechanical modulation. Importantly, our work demonstrates the complexities of d-orbitalbased interference effects that are not seen in any other carbon-based systems.

We study the conductance of two ferrocene derivatives consisting of a ferrocene unit terminated with two thioanisole gold-binding groups installed on the same $(\mathbf{1 , 3 - F e})$ or opposite (1,1'-Fe) Cp ring (Fig. 1a). We measure their single-molecule conductance using scanning tunneling microscope-based break-junction technique ${ }^{22,23}$ (see Methods for details) and compare the conductance features of the ferrocene derivatives to those of 4,4''-bis(methylthiol) p-terphenyl (P3, Fig. 1a, blue schematic molecular structure). Fig. 1b shows one-dimensional logarithmically binned conductance histograms created without data selection from 10,000 individual conductance-displacement traces. The conductances of $\mathbf{1 , 1} \mathbf{1}^{\prime}-\mathbf{F e}$ and $\mathbf{1 , 3 - F e}$ are $2 \times 10^{-5} \mathrm{G}_{0}$ and 3.3. $\times 10^{-5} \mathrm{G}_{0}$, respectively while the conductance of $\mathbf{P 3}$ is $1 \times 10^{-3} \mathrm{G}_{0}\left(\mathrm{G}_{0}=2 \mathrm{e}^{2} / \mathrm{h}\right.$, the conductance 
quantum). The two-dimensional conductance-displacement histograms clearly show that junctions of $\mathbf{P 3}$ sustain the longest elongation while those of 1,1'-Fe are the shortest (Fig. S1). This accounts for the different conductance peak heights as also corroborated by a histogram of plateau lengths (Fig. S2). Additionally, the ferrocene histogram peaks are broader near maximum and exhibit a tail that extends slightly beyond $10^{-3} \mathrm{G}_{0}$. We hypothesize that the conductance difference between $\mathbf{P 3}$ and the ferrocene derivatives as well as the difference in the histogram peak widths result from the larger range of junction geometries sampled by the ferrocene junctions due to the easy rotation around the $\mathrm{Cp}$ rings.
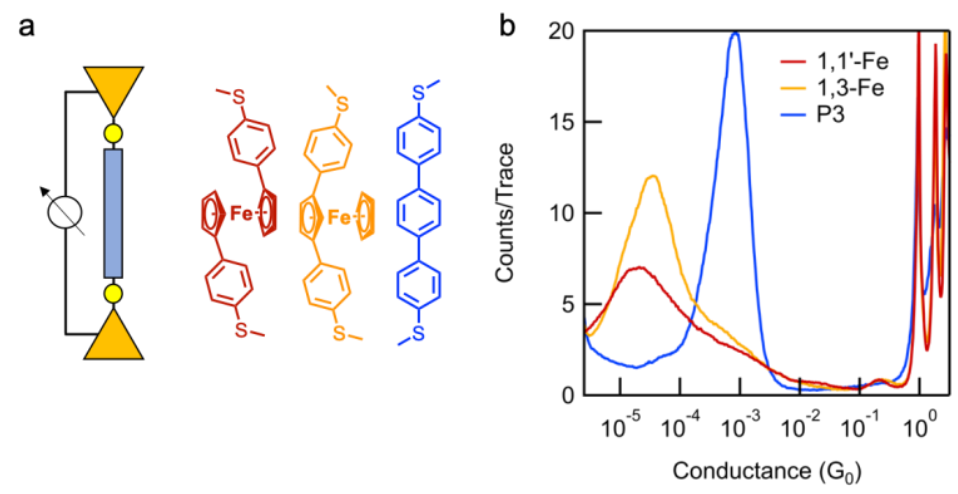

Figure 1. Molecule structures and conductance histograms. a. Junction schematic and molecular structures of 1,1'-Fe (red), 1,3-Fe (orange) and P3 (blue). b. Logarithmically binned onedimensional conductance histograms for 1,1'-Fe (red), 1,3-Fe (orange) and P3 (blue) measurements. All histograms were built from 10,000 conductance traces without data selection measured at an applied bias of $450 \mathrm{mV}$.

To test this hypothesis, we calculated the electronic transmission for model molecular junctions with all three systems (see Methods for the computational details). We show in Fig. 2a two views of the optimized junction with 1,1'-Fe. Analogous figures for 1,3-Fe and P3 are in Fig. S3. We plot the energy-dependent transmission functions, $T(E)$, calculated at zero-bias for all three molecules in Fig. 2b. Sharp anti-resonances indicative of destructive interference are observed between the Fermi energy $\left(E_{F}\right)$ and $-1 \mathrm{eV}$ for 1,3-Fe and 1,1'-Fe junctions, which suppress transmission around $\mathrm{E}_{\mathrm{F}}$. By contrast, the transmission function for $\mathbf{P 3}$ shows clear evidence of constructive interference between the molecular HOMO and LUMO, yielding a much higher transmission around $E_{F}$. Focusing on 1,1'-Fe junctions, we calculate its transmission for geometries with different relative angle between the two $\mathrm{Cp}$ rings (see inset of Fig. 3a and Fig. S4 for the angle definition). Transmission curves calculated for geometries with three different angles 
are shown in Fig. 3a. They clearly demonstrate that the anti-resonance and its position can be controlled by rotating one ring relative to the other.
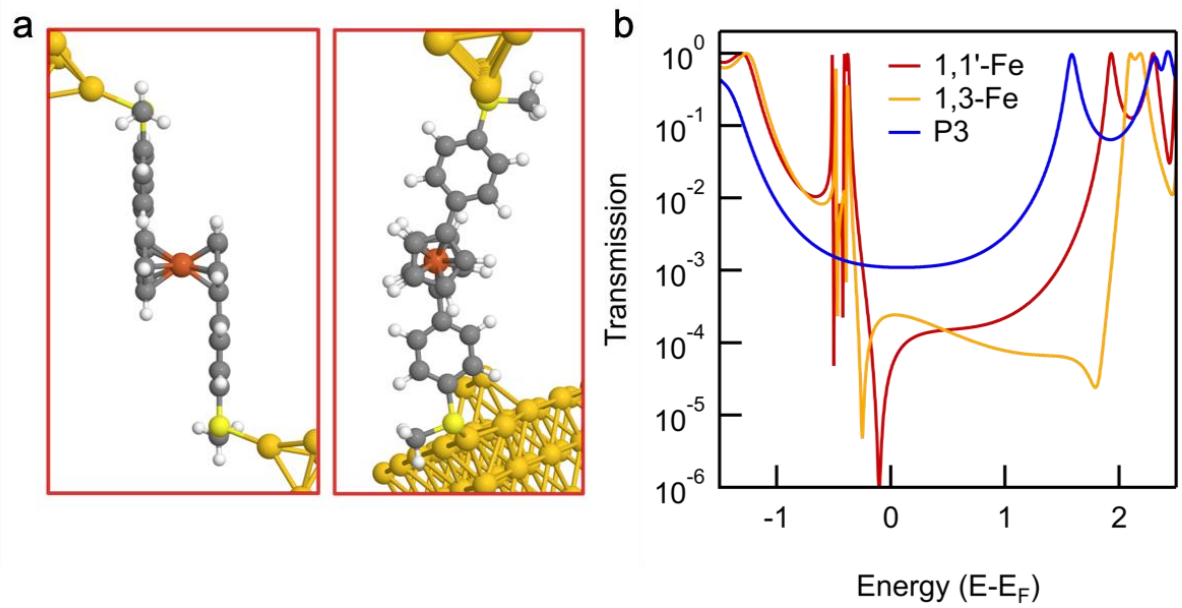

Figure 2: DFT-based calculations. a. Optimized molecular junction structure for 1,1'-Fe employed in the transport calculations. b. Transmission functions for 1,1'-Fe (red), 1,3-Fe (orange) and $\mathbf{P 3}$ (blue). Compared to $\boldsymbol{P 3}$, ferrocene-based transmissions show sharp antiresonances at around $E_{F}$, a signature of destructive quantum interference. By contrast, $\boldsymbol{P} 3$ shows evidence of constructive interference, producing a significant increase of the conductance at $E_{F}$.

To follow this evolution more carefully, we calculate $T(E)$ for a series of angles and compile these into a two-dimensional plot (Fig. 3b). The most prominent features are the blue regions corresponding to angular and energy intervals with strongly suppressed transmission (between $10^{-4}$ and $\left.10^{-7}\right)$. The sharp yellow lines of high transmission $\left(>10^{-1}\right)$ around $-0.5 \mathrm{eV}$ are resonances that are also seen in Fig. 3a. We attribute these to weakly coupled localized, ddominated molecular orbitals. At large angles $\left(\varphi>120^{\circ}\right)$, the two thioanisole phenyl rings begin to overlap and a broader blue low transmission feature appears as a result of direct tunneling between the thioanisole groups, bypassing the ferrocene unit. This is detailed in Fig. S5, where we see that the presence or absence of destructive interferences can change the conductance at $E_{F}$ by several orders of magnitude. Such geometries represent a very small S-S distance, and are highly unlikely to be probed in our experiments.

We analyze the contributions of the various orbitals of the system (molecule and electrode Au atoms) to the anti-resonances for 1,1'-Fe. In Fig. 3c, we show states at energies corresponding to the HOMO-3, HOMO and LUMO orbitals for the $30^{\circ}$ structure (see Fig. S6 for orbitals at other representative angles). These orbitals, which have contributions from the Fe d-states with $d_{\mathrm{yz}}, \mathrm{d}_{\mathrm{x} 2 \text { - }}$ 
y2, and $d_{x z}$ symmetries, are delocalized across the junctions as these d-states can couple to the Cp $\pi$-system. They give rise to the dominant transport resonances. By contrast, HOMO-2 (with $\mathrm{d}_{\mathrm{z} 2}$ symmetry) and HOMO-1 ( $\mathrm{d}_{\mathrm{xy}}$ symmetry) are strongly localized on the central ferrocene unit at all rotation angles because the overlap of hybridizing orbitals is small. They do not fit the nodal symmetry of the $\mathrm{Cp} \pi$-system. These orbitals manifest in the transmission as sharp resonances between $24^{\circ}$ and $120^{\circ}$ discussed above.

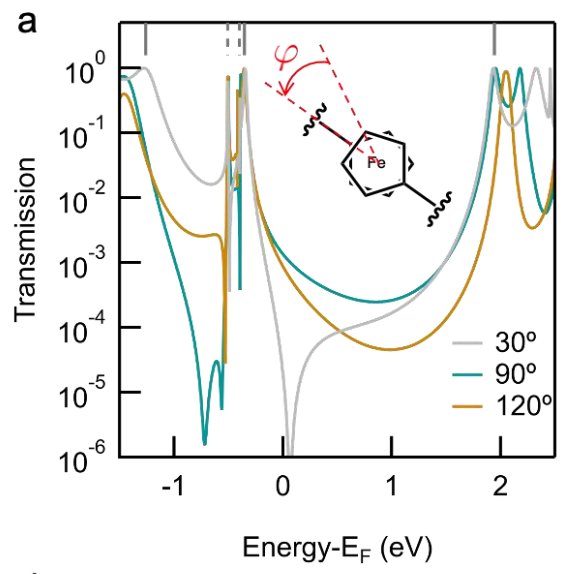

d

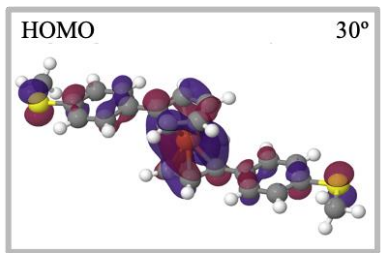

b

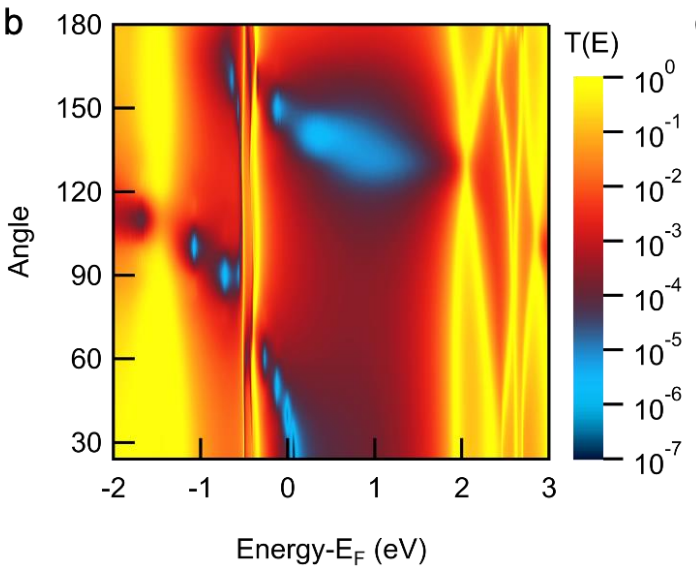

Energy- $E_{F}(e V)$

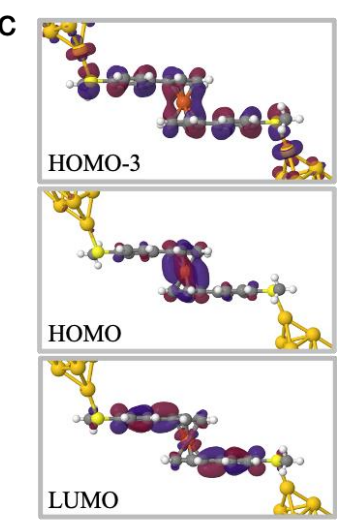

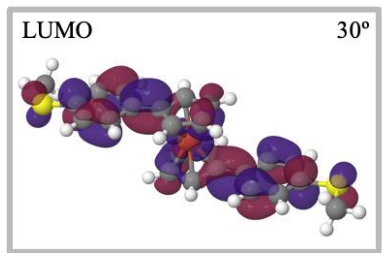
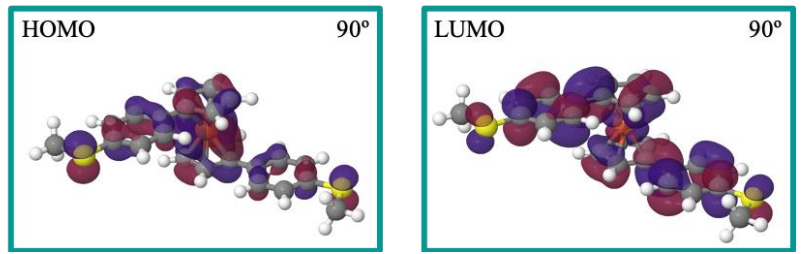

Figure 3: Transmission functions as a function of relative $C p$ ring angles for the 1,1'-Fe junctions. a. DFT-based transmission functions for 1,1'-Fe model junctions at 30 (grey), $90^{\circ}$ (green) and $120^{\circ}$ (gold). For 30 , an anti-resonance is seen between the HOMO and LUMO resonances. Coupled resonances (HOMO, HOMO-3, and LUMO) are marked by solid bar on the top axis while the dashed bars mark the uncoupled HOMO-1 and HOMO-2 resonance. At $90^{\circ}$, the antiresonance shifts to lower energies $(-0.8 \mathrm{eV})$. With an additional rotation of $30^{\circ}$, the interference is no longer destructive. Inset: Schematic illustrating angle definition, see Fig. S4 for further details. b. 2D-transmission map of 1,1'-Fe junctions for angles between $24^{\circ}$ and $180^{\circ}$. The areas associated to destructive quantum interference (blue) change with rotation. $c$. Scattering states at HOMO-3, HOMO and LUMO for the 30 1,1'-Fe junction. d. Gas-phase HOMO and LUMO of 1,1'-Fe for $30^{\circ}$ and $90^{\circ}$ angle. The nodal structure of the HOMO changes going from $30^{\circ}$ to $90^{\circ}$.

In order to explain how rotating the $\mathrm{Cp}$ rings modifies interferences, we generalize results of previous authors. ${ }^{24-29}$ Our argument embarks on the familiar trace formula for the transmission $T(E)=\operatorname{Tr}\left(\Gamma_{L} G \Gamma_{R} G^{+}\right) \cdot{ }^{30}$ It expresses $T(E)$ as the product of two Greens functions, $G(E)$ which 
are sums of a series of poles, where each pole represents a molecular orbital. ${ }^{31}$ We thus have a representation

$$
T(E)=\sum_{i} T_{i}+\sum_{i \neq j} T_{i j}
$$

The first term in Eq. 1 sums the single-pole contributions which are well parameterized as Lorentzians

$$
T_{i}(E)=\frac{\gamma_{i L} \gamma_{i R}}{\left(E-\varepsilon_{i}\right)^{2}+\gamma_{i}^{2}}
$$

where $\gamma_{i\{L, R\}}$ denotes the broadening resulting from the coupling of the $i^{\text {th }}$ orbital to left/right electrodes and $\gamma_{i}=\left(\gamma_{i L}+\gamma_{i R}\right) / 2$. The second term in Eq. 1 includes the mixed terms of the product, i.e. the interference terms which are effectively:

$$
T_{i j}(E) \cong-2 \cos \psi_{i j}\left|T_{i}(E)\right|^{\frac{1}{2}}\left|T_{j}(E)\right|^{\frac{1}{2}} .
$$

The angle $\psi_{i j}$ accounts for the relative phase of the tunneling wave across the orbitals $i$ and $j$. For well separated resonances, $\gamma_{i}, \gamma_{j} \ll\left|E_{i}-E_{j}\right|, T_{i j}(E)$ is small irrespective of the type of molecule considered. When $\psi_{i j}=\pi$, interference is constructive, while at $\psi_{i j}=0$ it is destructive if $E_{i}<$ $E<E_{j} .{ }^{24}$ Importantly the relative phase $\psi_{i j}$ is a property inherent to the molecule and thus can be inferred by looking at the molecular orbitals of the isolated molecule as long as the orbitals do not reorder when the molecule is coupled to the electrodes. Indeed, for the molecule with $\mathrm{Cp}$ rings at $30^{\circ}$ relative angle, the amplitude of both the HOMO and LUMO on the left and right $\mathrm{S}$ atom have the opposite sign, hence the relative shift vanishes, $\psi_{i j} \sim 0$ and the interference is destructive (Fig. $3 \mathrm{~d}$ ). For a $90^{\circ}$ relative angle, the amplitude of the HOMO is the same on each $\mathrm{S}$ atom, making the relative phase-shift $\psi_{i j} \approx \pi$. The interference is constructive and leads to a transmission with no antiresonance between the HOMO and LUMO resonances, as observed in Fig. 3a. Similar analyses can be made with other pairs of orbitals. For example, HOMO and HOMO-3 show evidence of constructive interference in the $30^{\circ}$ junction for an energy range between the two orbitals.

To experimentally demonstrate this variation in transmission with the rotation angle, we carry out modified STM-BJ experiments with 1,1'-Fe where we first form a molecular junction, then modulate the tip-sample separation with an amplitude of $2.4 \AA$ while measuring the conductance (Fig. 4a). The distance of $2.4 \AA$ was chosen as this is close to the change in the calculated junction length going from $30^{\circ}$ to $90^{\circ}$. In Fig. $4 \mathrm{~b}$, we overlay all traces that form and 
sustain a molecular junction during the modulation in a two-dimensional histogram (1900 out of 4300 traces). We find that as the tip-sample separation decreases, the junction conductance increases significantly. The inset of Fig. $4 \mathrm{~b}$ shows line profiles comparing the conductances when the junctions are pushed together or pulled apart during the first hold segment. The conductance increases on average by a factor of 6.7 when pushed together by $2.4 \AA$. By contrast, when the same experiment is performed with P3, the conductance increases by a factor of only 2.6. Although the highest conductance measured for $\mathbf{1 , 1}$ '-Fe is still lower than that of $\mathbf{P 3}$, the fact that the conductance for 1,1'-Fe has a higher modulation ratio with changing distance strongly supports that the 1,1'-Fe junctions are conformationally more flexible, and that such conformational changes have a greater influence on 1,1'-Fe junction conductance.

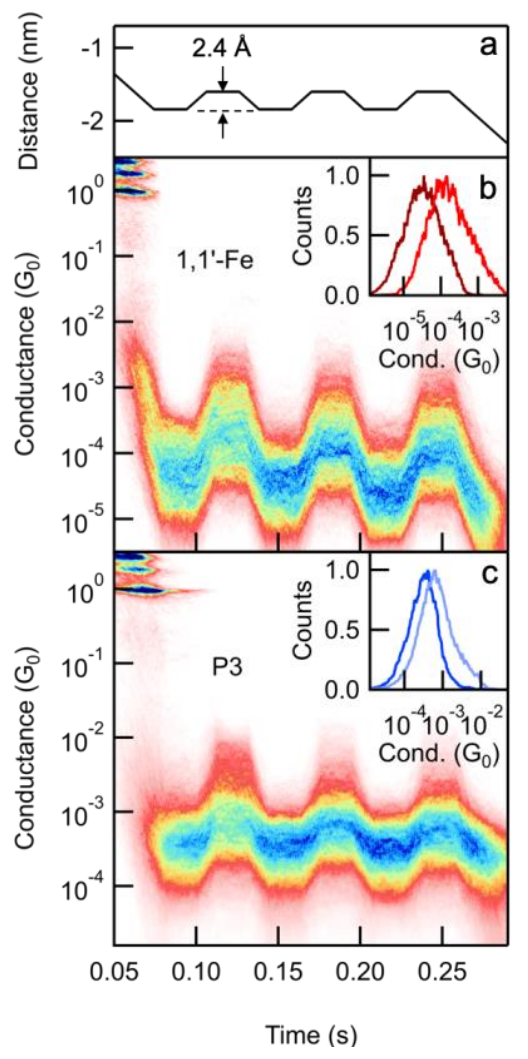

Figure 4: Modulation of the tip/sample separation in 1,1'-Fe and P3 junctions. a. Piezo displacement as a function of time showing the modulation of the tip/substrate gap. $b .2 D$ histogram of 1,1'-Fe traces obtained during tip/sample modulation. The conductance of the junction increases (decreases) when the distance between tip and sample is decreased (increased). The conductance changes by a factor of $\sim 7$ upon modulation. Inset: Line-profiles taken at $\sim 0.09 \mathrm{~s}$ (dark) and $0.12 \mathrm{~s}$ (light) comparing the conductance between the maximal and minimal elongation point. c. Analogous data for P3 showing that the conductance increase upon modulation is only a factor $\sim 3$. 
We have shown that molecular junctions comprising ferrocene derivatives with different substitution patterns exhibit destructive quantum interference effects produced by the interplay of several localized and delocalized orbitals. These interferences determine the transmission characteristics and decrease the low-bias conductance while allowing the conductance to be changed by modulating the electrode separation. Our single-molecule conductance measurements are corroborated by theoretical transmission computations showing how interference features of the ferrocene derivative (from destructive to constructive) evolve when the $\mathrm{Cp}$ rings rotate relative to one another, a low-energy conformational degree of freedom inherent to ferrocene.

\section{Methods.}

STM-BJ Measurements: We measure the single-molecule conductance using the STM-BJ technique with a custom-built setup described previously ${ }^{26}$. Briefly, we drive a Au tip in and out of contact with a Au-substrate and record the junction's conductance as the tip is withdrawn. Upon rupture of the Au contact, a molecule may bridge the gap as evidenced by an additional plateau in the conductance versus displacement trace. We collect 10,000 such traces, which contain 2,000 data points per nanometer of extension (40 kHz sampling rate) and construct the (normalized) 1D and 2D conductance histograms without data selection. All ferrocene derivatives studied here were introduced into the setup in a tetradecane solution with $0.1 \sim 1 \mathrm{mM}$ concentration while P3 was studied from a 1,2,4-trichlorobenzene solution.

DFT Calculations: Molecular junctions consist of molecules bound to pyramidal Au electrodes; geometries are optimized using density functional theory (DFT) employing FHI-aims ${ }^{32}$ with PBE functional. $^{33}$ Transport computations use DFT with the non-equilibrium Green's function formalism (NEGF) as implemented in the package AITRANSS ${ }^{34,35}$; details see Sec. III in SI.

Acknowledgements. M.C.-G. and D.H.-P. thank R. Korytár for discussions. M.C.-G., D. H.-P. and F. E. acknowledge financial support from the German Research Foundation (DFG) through Research Training Group (GRK) 1570 and Collaborative Research Center (SFB) 1277 - Project ID 314695032 (subprojects A03, B01). L.V., X.R. and G. L. acknowledge support by the Center for Precision Assembly of Superstratic and Superatomic Solids at Columbia University, an NSF MRSEC (award number DMR-1420634) and by NSF CHE-1807654. M.S.I. was supported by a 
Marie Skłodowska Curie Global Fellowship (MOLCLICK: 657247) within the Horizon 2020 Programme.

\section{References.}

1 Kealy, T.J. \& Pauson, P.L., A new type of organo-iron compound. Nature 168, 1039-1040 (1951).

2 Miller, S.A., Tebboth, J.A., \& Tremaine, J.F., 114. Dicyclopentadienyliron. Journal of the Chemical Society (Resumed), 632-635 (1952).

3 Astruc, D., Why is ferrocene so exceptional? European Journal of Inorganic Chemistry 2017, 6-29 (2017).

4 Togni, A. \& Hayashi, T., Ferrocenes: Homogeneous catalysis, organic synthesis, materials science. (Wiley-VCH, Weinheim, 1995).

5 Haaland, A. \& Nilsson, J.-E., The determination of the barrier to internal rotation in ferrocene and ruthenocene by means of electron diffraction. Chemical Communications (London), 88-89 (1968).

6 Abel, E.W., Long, N.J., Orrell, K.G., Osborne, A.G., \& Šik, V., Dynamic nmr studies of ring rotation in substituted ferrocenes and ruthenocenes. J. Organomet. Chem. 403, 195208 (1991).

7 Vollmann, M. \& Butenschön, H., Synthesis of a functionalized dialkynylferrocene for molecular electronics. Comptes Rendus Chimie 8, 1282-1285 (2005).

8 Getty, S.A. et al., Near-perfect conduction through a ferrocene-based molecular wire. Phys. Rev. B 71, 241401 (2005).

9 Nijhuis, C.A., Reus, W.F., \& Whitesides, G.M., Mechanism of rectification in tunneling junctions based on molecules with asymmetric potential drops. J. Am. Chem. Soc. 132, 18386-18401 (2010).

10 Matsuura, Y., Current rectification in nickelocenylferrocene sandwiched between two gold electrodes. J. Chem. Phys. 138, 014311 (2013).

11 Liu, R., Ke, S.-H., Yang, W., \& Baranger, H.U., Cobaltocene as a spin filter. J. Chem. Phys. 127, 141104 (2007). 
12 Zhou, L. et al., One-dimensional iron-cyclopentadienyl sandwich molecular wire with half metallic, negative differential resistance and high-spin filter efficiency properties. J. Am. Chem. Soc. 130, 4023-4027 (2008).

13 Pal, A.N. et al., Nonmagnetic single-molecule spin-filter based on quantum interference. Nat. Commun. 10, 5565 (2019).

14 Zhao, X., Kastlunger, G., \& Stadler, R., Quantum interference in coherent tunneling through branched molecular junctions containing ferrocene centers. Phys. Rev. B 96 (2017).

15 Zhao, X. \& Stadler, R., Dft-based study of electron transport through ferrocene compounds with different anchor groups in different adsorption configurations of an stm setup. Phys. Rev. B 99, 045431 (2019).

16 Kanthasamy, K. et al., Charge transport through ferrocene 1,1'-diamine single-molecule junctions. Small 12, 4849-4856 (2016).

17 Bredow, T. et al., Ferrocene-1,1'-dithiol as molecular wire between ag electrodes: The role of surface defects. J. Chem. Phys. 128, 064704 (2008).

18 Morari, C. et al., Electronic transport properties of 1,1'-ferrocene dicarboxylic acid linked to al(111) electrodes. ACS Nano 3, 4137-4143 (2009).

19 Joy, J., Stuyver, T., \& Shaik, S., Oriented external electric fields and ionic additives elicit catalysis and mechanistic cross-over in oxidative addition reactions. J. Am. Chem. Soc. (2020).

20 Zhu, T., Jiménez-Hoyos, C.A., McClain, J., Berkelbach, T.C., \& Chan, G.K.-L., Coupledcluster impurity solvers for dynamical mean-field theory. Phys. Rev. B 100, 115154 (2019).

21 Low, J.Z. et al., The anomalous behavior of the blatter radical at the metal-molecule interface. (in preparation).

22 Xu, B.Q. \& Tao, N.J., Measurement of single-molecule resistance by repeated formation of molecular junctions. Science 301, 1221-1223 (2003).

23 Venkataraman, L. et al., Single-molecule circuits with well-defined molecular conductance. Nano Lett. 6, 458 - 462 (2006).

24 Yoshizawa, K., Tada, T., \& Staykov, A., Orbital views of the electron transport in molecular devices. J. Am. Chem. Soc. 130, 9406-9413 (2008). 
25 Taniguchi, M. et al., Dependence of single-molecule conductance on molecule junction symmetry. J. Am. Chem. Soc. 133, 11426-11429 (2011).

26 Géranton, G., Seiler, C., Bagrets, A., Venkataraman, L., \& Evers, F., Transport properties of individual c60-molecules. J. Chem. Phys. 139, 234701 (2013).

27 Datta, S. et al., Novel interference effects between parallel quantum wells. Phys. Rev. Lett. 55, 2344-2347 (1985).

28 Solomon, G.C. et al., Understanding quantum interference in coherent molecular conduction. J. Chem. Phys. 129, - (2008).

29 Gunasekaran, S., Greenwald, J.E., \& Venkataraman, L., Visualizing quantum interference in molecular junctions. Nano Lett. (2020).

30 Jauho, A.P., Wingreen, N.S., \& Meir, Y., Time-dependent transport in interacting and noninteracting resonant-tunneling systems. Physical review. B, Condensed matter 50, 5528-5544 (1994).

31 Evers, F., Korytár, R., Tewari, S., \& van Ruitenbeek, J.M., Advances and challenges in single-molecule electron transport. arXiv e-prints, arXiv:1906.10449 (2019).

32 Blum, V. et al., Ab initio molecular simulations with numeric atom-centered orbitals. Comp. Phys. Commun. 180, 2175-2196 (2009).

33 Perdew, J.P., Burke, K., \& Ernzerhof, M., Generalized gradient approximation made simple. Phys. Rev. Lett. 77, 3865-3868 (1996).

34 Arnold, A., Weigend, F., \& Evers, F., Quantum chemistry calculations for molecules coupled to reservoirs: Formalism, implementation, and application to benzenedithiol. $J$. Chem. Phys. 126, 174101 (2007).

35 Bagrets, A., Spin-polarized electron transport across metal-organic molecules: A density functional theory approach. J. Chem. Theory Comput. 9, 2801-2815 (2013). 


\title{
Supplementary Material
}

\section{Mechanically-Tunable Quantum Interference in Ferrocene-based Single-Molecule Junctions}

\author{
María Camarasa-Gómez, ${ }^{1}$ Daniel Hernangómez-Pérez, ${ }^{1,2}$ Michael S. Inkpen, ${ }^{3}$ Giacomo \\ Lovat, ${ }^{3}$ E-Dean Fung, ${ }^{3}$ Xavier Roy, ${ }^{4}$ Latha Venkataraman, ${ }^{3,4}$ and Ferdinand Evers ${ }^{1}$ \\ ${ }^{1}$ Institute of Theoretical Physics, University of Regensburg, 93040 Regensburg, Germany \\ ${ }^{2}$ Department of Materials and Interfaces, \\ Weizmann Institute of Science, Rehovot 761001, Israel \\ ${ }^{3}$ Department of Applied Physics and Applied Mathematics, Columbia University, \\ New York, New York 10027, United States \\ ${ }^{4}$ Department of Chemistry, Columbia University, \\ New York, New York 10027, United States
}

Email: inkpen@usc.edu; lv2117@columbia.edu; ferdinand.evers@ur.de;

\section{CONTENTS}

Synthetic details

NMR spectra 


\section{SYNTHETIC DETAILS}

All reactions were conducted in oven-dried glassware under a nitrogen or argon atmosphere, though no special precautions were taken to exclude air or moisture during workup and purification. The preparation of 1,3-bis(tri[n-butyl]stannyl)ferrocene followed literature methods.[1] Stannylated precursors were purified using alumina, Brockmann grade II. Stille[2, 3] and Negishi[4, 5] conditions were based on previous reports. Zinc chloride and cesium fluoride were dried immediately prior to use by heating under vacuum at $140^{\circ} \mathrm{C}$ overnight[5] or at $100^{\circ} \mathrm{C}$ for $2.5 \mathrm{~h}[6]$, respectively. Anhydrous and deoxygenated THF was prepared by elution through a dual-column solvent system (MBraun SPS). All other materials were commercially available and used as received. Reported methods are unoptimized, with some yields also impacted by closely eluting impurities (particularly in the isolation of 1,3-Fe) that reduced the efficiency of purification attempts using column chromatography.

${ }^{1} \mathrm{H}$ and ${ }^{13} \mathrm{C}\left\{{ }^{1} \mathrm{H}\right\}$ NMR spectra were recorded on a Bruker Avance III 400, 400SL (400 $\mathrm{MHz})$, or $500(500 \mathrm{MHz})$ spectrometer and referenced to the residual solvent peaks of $\mathrm{CD}_{2} \mathrm{Cl}_{2}$ at $\delta 5.32\left({ }^{1} \mathrm{H}\right)$ and $53.84\left({ }^{13} \mathrm{C}\left\{{ }^{1} \mathrm{H}\right\}\right)$ ppm. Mass spectrometry analyses were conducted by Brandon Fowler of the Mass Spectrometry Facility, Chemistry Department, Columbia University, New York using a Waters XEVO G2-XS QToF mass spectrometer.

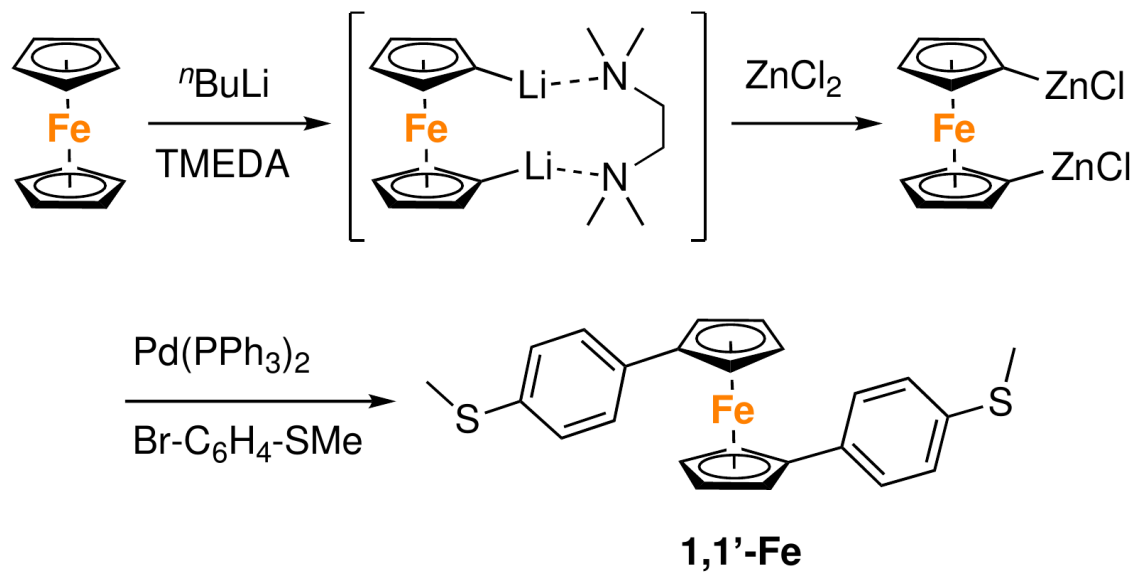

Scheme 1. Synthetic route to $\mathbf{1 , 1}$ '-Fe (TMEDA = N,N,N,N'-tetramethylethanediamine).

\section{Synthesis of 1,1'-Fe:}

TMEDA (1.66 mL, $11.1 \mathrm{mmol})$ and $2.5 \mathrm{M} \mathrm{n}$-BuLi in hexanes (4.76 mL, $11.9 \mathrm{mmol})$ were added to a stirred suspension of ferrocene $(1.006 \mathrm{~g}, 5.407 \mathrm{mmol})$ in n-pentane $(20 \mathrm{~mL})$ held 
at $0^{\circ} \mathrm{C}$ (ice bath). After stirring overnight to room temperature, the majority of solvent was removed by cannula filtration and the orange slurry dissolved in THF $(16 \mathrm{~mL})$ at $0^{\circ} \mathrm{C}$. A freshly prepared solution of $\sim 0.97 \mathrm{M} \mathrm{ZnCl}$ in THF $(11.4 \mathrm{~mL}, \sim 11.1 \mathrm{mmol})$ was added, and the mixture was stirred at room temperature for $2.5 \mathrm{~h}$. $\mathrm{Pd}\left(\mathrm{PPh}_{3}\right)_{4}(0.756 \mathrm{~g}, 0.654$ mmol) and 4-bromothioanisole (2.419 g, $11.91 \mathrm{mmol})$ were added against nitrogen and the reaction was stirred at $60^{\circ} \mathrm{C}$ for $18 \mathrm{~h}$. After cooling to room temperature, solvent was removed in vacuo. The crude product was pre-absorbed on $\mathrm{SiO}_{2}$ and purified by column chromatography, eluting with $\mathrm{CH}_{2} \mathrm{Cl}_{2}$-hexanes $(0: 100 \rightarrow 30: 70 \mathrm{v} / \mathrm{v}) \cdot \mathbf{1 , 1}$ '-Fe was isolated as an orange solid from fractions of the second major orange band (0.961 g, 41\%).

${ }^{1} \mathrm{H}$ NMR $\left(400 \mathrm{MHz}, \mathrm{CD}_{2} \mathrm{Cl}_{2}\right.$ ): $\delta(\mathrm{ppm}) 2.49$ (s, 6H, -SCH 3 ), 4.21 (pseudo-t, $4 \mathrm{H}, J=1.87$ Hz, Cp- $H$ ), 4.46 (pseudo-t, 4H, $J=1.87$ Hz, Cp- $H$ ), 7.05 (m, 4H, Ar- $H$ ), 7.18 (m, 4H, Ar- $H) .{ }^{13} \mathrm{C}\left\{{ }^{1} \mathrm{H}\right\}$ NMR $\left(101 \mathrm{MHz}, \mathrm{CD}_{2} \mathrm{Cl}_{2}\right): \delta(\mathrm{ppm}) 16.19\left(2 \mathrm{C},-\mathrm{S} C \mathrm{H}_{3}\right), 67.91(4 \mathrm{C}, \mathrm{Cp} C-$ H), 70.74 (4C, Cp C-H), 86.15 (2C, Cp C-Ar), 126.66 (4C, Ar C-H), 126.94 (4C, Ar C-H), 135.19 (2C, Ar $C$-R), 136.02 (2C, Ar $C$-R). HRMS (ASAP+): m/z $431.0590\left([\mathrm{M}+\mathrm{H}]^{+}\right.$calc. for $\mathrm{C}_{24} \mathrm{H}_{23} \mathrm{FeS}_{2}$ : 431.0591).

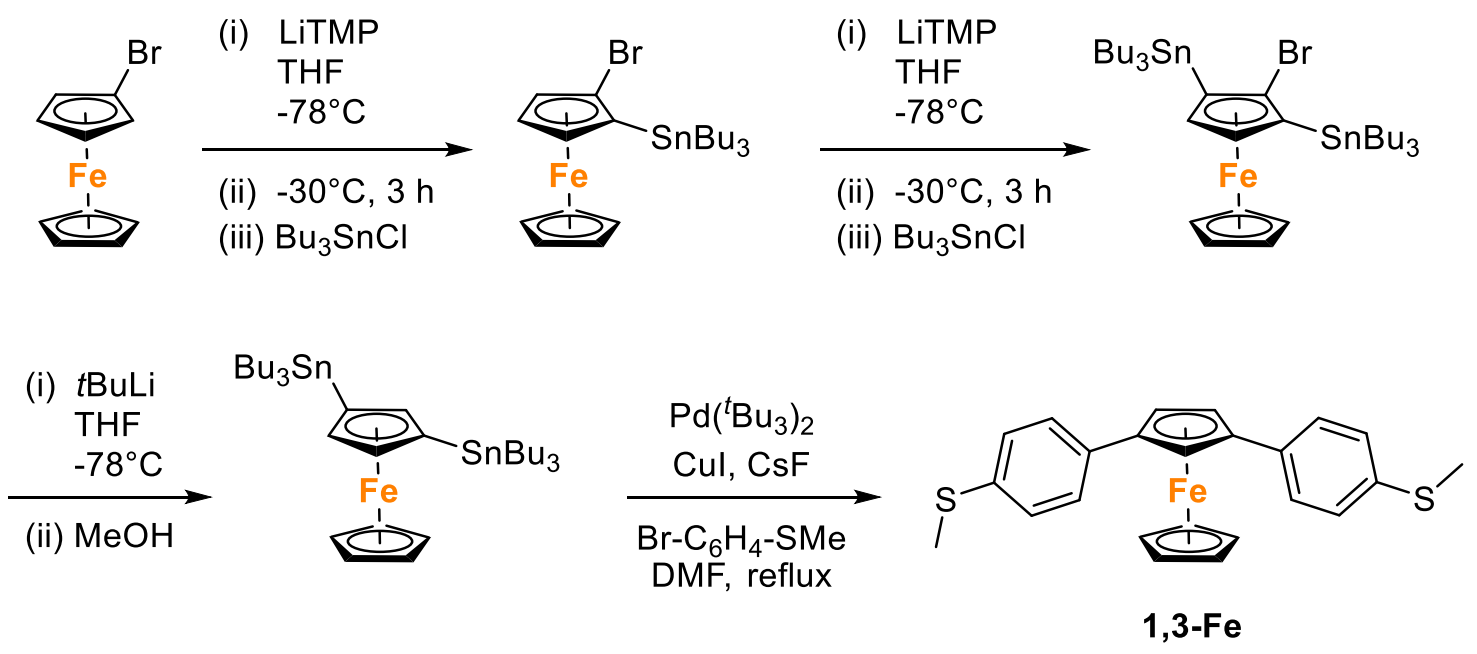

Scheme 2. Synthetic route to $\mathbf{1 , 3 - F e}$.

\section{Synthesis of 1,3-Fe:}

1,3-Bis(tri[n-butyl]stannyl)ferrocene (0.361 g, $0.472 \mathrm{mmol})$, 4-bromothioanisole (0.229 g, $1.13 \mathrm{mmol}), \mathrm{CuI}(0.009 \mathrm{~g}, 0.05 \mathrm{mmol}), \mathrm{Pd}\left(\mathrm{t}-\mathrm{Bu}_{3}\right)_{2}(0.012 \mathrm{~g}, 0.023 \mathrm{mmol})$ and $\mathrm{DMF}(3 \mathrm{~mL})$ were added against nitrogen to dry $\mathrm{CsF}(\sim 0.184 \mathrm{~g}, \sim 1.21 \mathrm{mmol})$, and stirred at $100^{\circ} \mathrm{C}$ for $22 \mathrm{~h}$. The cooled mixture was extracted into $\mathrm{CH}_{2} \mathrm{Cl}_{2}$ and $\mathrm{H}_{2} \mathrm{O}$, whereby the organic layer was filtered through Celite. After solvent removal the crude solid material was pre- 
absorbed on $\mathrm{SiO}_{2}$ and purified by column chromatography, eluting with $\mathrm{CH}_{2} \mathrm{Cl}_{2}$-hexanes $(0: 100 \rightarrow 20: 80 \mathrm{v} / \mathrm{v})$. $\mathbf{1 , 3 - F e}$ was isolated as an orange solid from selected fractions of the third orange band $(0.006 \mathrm{~g}, 3 \%)$.

${ }^{1} \mathrm{H}$ NMR $\left(400 \mathrm{MHz}, \mathrm{CD}_{2} \mathrm{Cl}_{2}\right): \delta(\mathrm{ppm}) 2.50\left(\mathrm{~s}, 6 \mathrm{H},-\mathrm{SCH}_{3}\right), 3.90(\mathrm{~s}, 5 \mathrm{H}, \mathrm{Cp}-H), 4.79$ $(\mathrm{d}, 2 \mathrm{H}, J=1.47 \mathrm{~Hz}, \mathrm{Cp}-H), 5.12(\mathrm{t}, 1 \mathrm{H}, J=1.47 \mathrm{~Hz}, \mathrm{Cp}-\mathrm{H}), 7.22(\mathrm{~m}, 4 \mathrm{H}$, Ar- $H), 7.48$ $(\mathrm{m}, 4 \mathrm{H}, \mathrm{Ar}-H) \cdot{ }^{13} \mathrm{C}\left\{{ }^{1} \mathrm{H}\right\} \operatorname{NMR}\left(101 \mathrm{MHz}, \mathrm{CD}_{2} \mathrm{Cl}_{2}\right): \delta(\mathrm{ppm}) 16.13\left(2 \mathrm{C},-\mathrm{SCH}_{3}\right), 64.91(1 \mathrm{C}$, Cp $C$-H), 67.46 (2C, Cp C-H), 71.57 (5C, Cp C-H), 86.17 (2C, Cp C-Ar), 126.83 (4C, Ar $C$-H), 127.04 (4C, Ar $C$-H), 136.24 (2C, Ar $C$-R), 136.33 (2C, Ar $C$-R). HRMS (ASAP+): $\mathrm{m} / \mathrm{z} 431.0587\left([\mathrm{M}+\mathrm{H}]^{+}\right.$calc. for $\mathrm{C}_{24} \mathrm{H}_{23} \mathrm{FeS}_{2}$ : 431.0591)

\section{MEASUREMENT DETAILS}

We measured the conductance of single-molecule junctions formed with two gold electrodes using a custom-built modified Scanning Tunneling Microscope (STM).[7] We used $0.25 \mathrm{~mm}$ diameter cut gold wire (99.95\%, Alfa Aesar) as the STM tip and $100 \mathrm{~nm}$ goldcoated (99.999\%, Alfa Aesar) steel pucks as substrates. A commercially available single-axis piezoelectric positioner (Nano-P15, Mad City Labs) was used to achieve sub-angstrom level control of the tip-substrate distance. The STM was controlled using a custom written program in IgorPro (Wavemetrics, Inc.) and operated in ambient conditions at room temperature. The gold substrates were cleaned using a UV/ozone cleaning lamp for 20 minutes prior to use. For each measurement, 1000 traces were first collected prior to adding molecular solutions to check the cleanliness of the gold surface. Solutions of the target molecules at $100 \mathrm{M}$ concentration in tetradecane (Alfa Aesar, 99\%purity) were added to the substrate for STM break-junction measurements. After the formation of each Au-Au junction with a conductance greater than $5 \mathrm{G}_{0}$, the piezoelectric positioner moved the substrate at a speed of $20 \mathrm{~nm} / \mathrm{s}$ to break the junction. The current and voltage across the junction were acquired at $40 \mathrm{kHz}$.

\section{$A B-I N I T I O$ CALCULATIONS DETAILS}

Ab-initio calculations were carried out using the closed-shell Kohn-Sham formulation of density functional theory implemented in the FHI-aims code[8]. A non-empirical general- 
ized gradient-corrected approximation (PBE) for the exchange-correlation functional[9] was considered. Scalar relativistic corrections to the kinetic energy were incorporated in our firstprinciples calculations at the atomic zeroth-order regular approximation (ZORA) level[10]. The Kohn-Sham states were represented in an optimized all-electron numeric atom-centered basis set ('tight' computational settings, roughly equivalent to "double zeta + polarization" quality for the molecular atoms and "double zeta quality for the gold atoms which belong to the electrodes). The ground state was obtained using standard convergence criteria in the self-consistent field cycle for the difference in the particle density $\left(10^{-5}\right.$ a.u.), total energy $\left(10^{-6} \mathrm{eV}\right)$, sum of Kohn-Sham eigenvalues $\left(10^{-4} \mathrm{eV}\right)$ and forces $\left(10^{-4} \mathrm{eV} / \AA\right)$.

The geometries of the molecular junctions were obtained using a well-established two-step process. In the first step, the positions of the molecular atoms and the tip of the electrodes were optimized using the trust-radius enhanced version of the Broyden-Fletcher-ShannoGoldfarb algorithm[8]. The pyramidal gold clusters used for this geometry optimization contain up to 11 gold atoms per pyramid. Based on test optimizations, we also constrain the S-Me dihedral angle so that the methyl group lies in the same plane as the functionalized phenyl rings. In the second step, the resulting molecular and tip geometries were fixed and additional layers of gold atoms added to the external planes of the previously optimized electrode tips to ensure screening of the excess charge (and therefore, correct level alignment) in the subsequent quantum transport calculations. All the molecular and junction geometries studied in this work were considered to be structurally relaxed when no force component per atom exceeded the threshold value of $10^{-2} \mathrm{eV} / \AA$.

The energy-dependent electronic transmissions were calculated in the regime of linear response using the non-equilibrium Green's function formalism as implemented in the transport package AITRANSS[11, 12]. Each junction electrode was modeled by a pyramidal cluster of 55 atoms with closest interatomic distance of $2.88 \AA$. The latter cluster was cut along the (111) direction. The self-energy of the reservoirs was parametrized by an energyindependent (Markovian) spatially local function, $\Sigma\left(\mathbf{r}, \mathbf{r}^{\prime}\right)=i \eta(\mathbf{r}) \delta\left(\mathbf{r}-\mathbf{r}^{\prime}\right)$ with non-zero values only in the subspace associated to the outermost layers of the finite cluster. The local absorption rate, $\eta(\mathbf{r})$, was adjusted to guarantee that the electronic transmission remained invariant under smooth moderate changes in $\eta(\mathbf{r})$. 


\section{ADDITIONAL DATA}
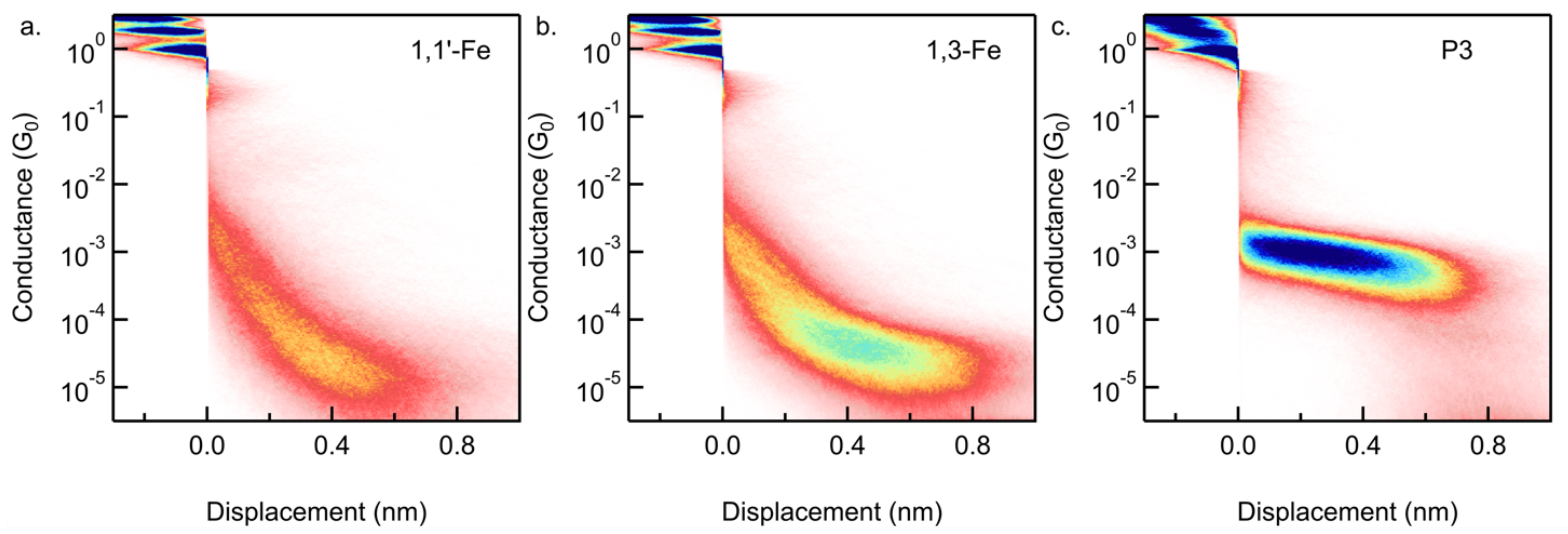

Fig. S1. Two-dimensional logarithmically-binned histograms for a. 1,1'-Fe, b. 1,3-Fe and c. P3. Each histogram is compiled from 10000 traces without data selection. Histograms have 200 bins per nm along the $\mathrm{x}$-axis and 89 bins per decade along the $\mathrm{y}$-axis

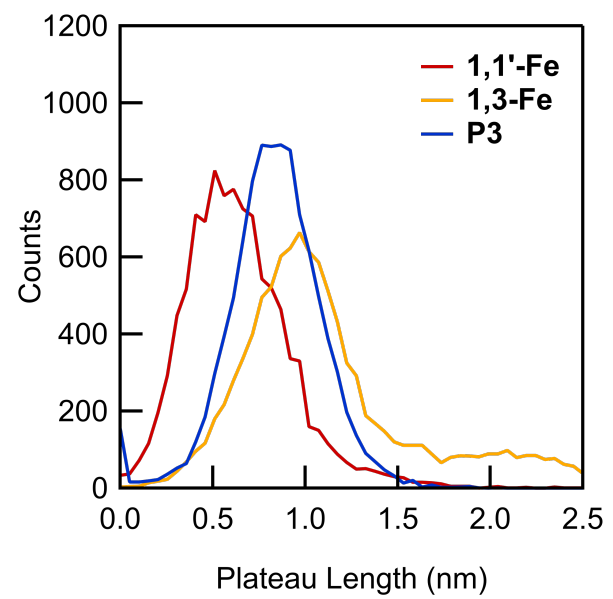

Fig. S2. Plateau length histograms determined by analyzing 10,000 traces for each molecule. For 1,1'-Fe and 1,3-Fe, plateau lengths were analyzed between $4 \times 10^{-5}$ and $10^{-3} \mathrm{G}_{0}$. For $\mathbf{P 3}$, plateau lengths were analyzed between $4 \times 10^{-4}$ and $10^{-2} \mathrm{G}_{0}$. These limits were determined from the 1D histograms shown in Fig.1b. 

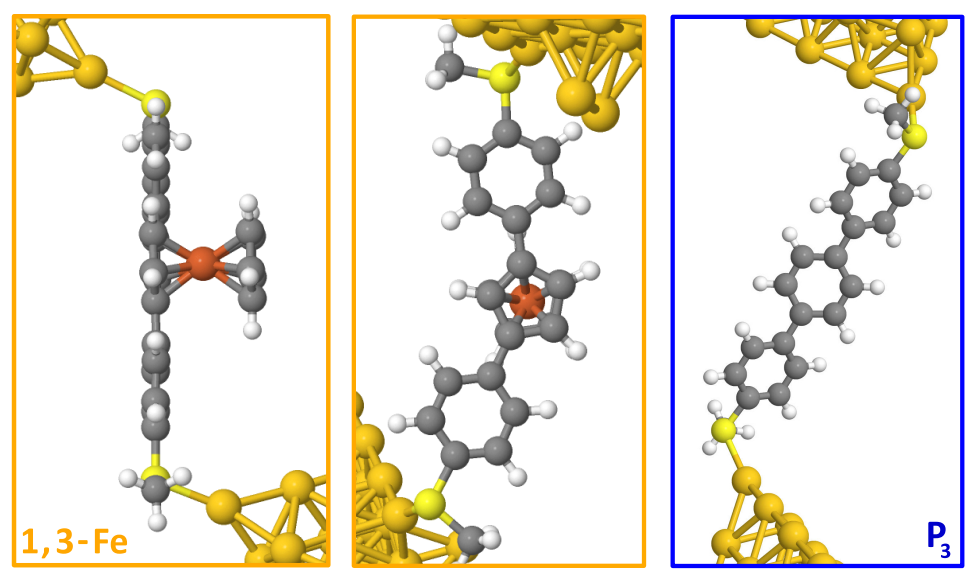

Fig. S3. Optimized junction geometries of 1,3-Fe (side and top view) and P3 (top view) used for the quantum transport calculations presented in Fig. $2 \mathrm{~b}$ of the main paper.

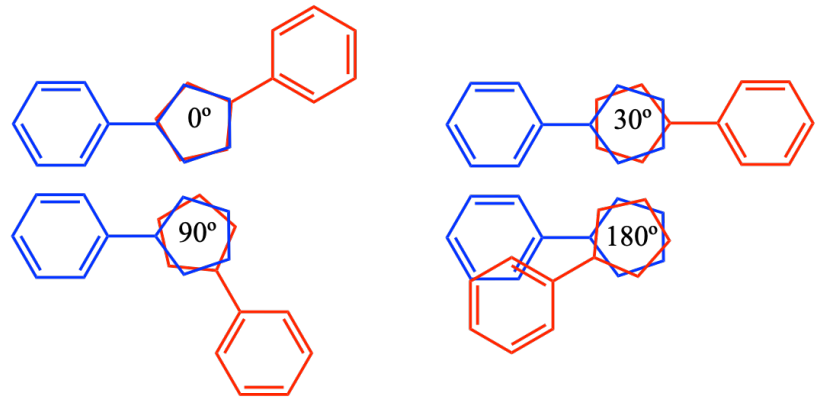

Fig. S4. 1,1'-Fe with different relative angles (SMe and Fe not shown). Note that $0^{\circ}$ is the relaxed geometry.
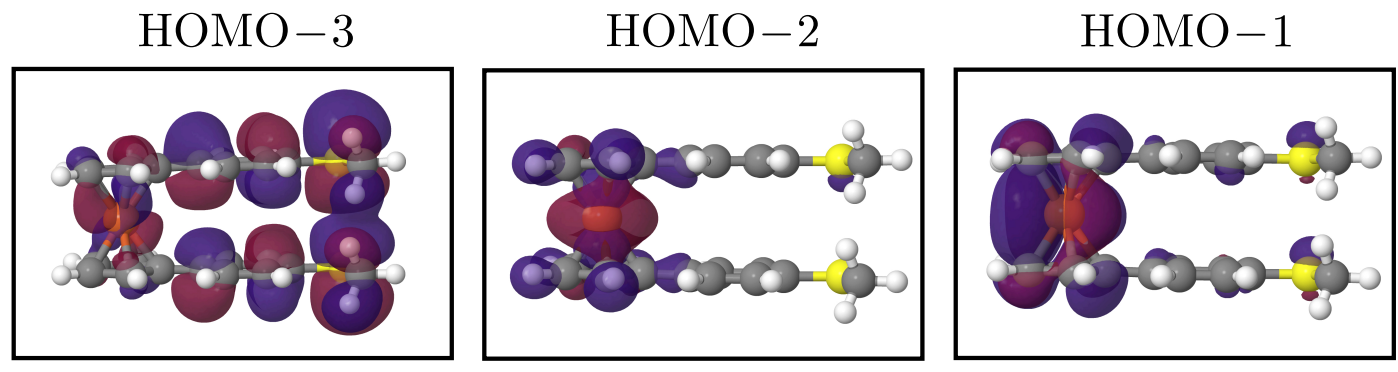

Fig. S5. Isosurface plot of the Kohn-Sham orbitals HOMO-3, HOMO-2 and HOMO-1 of the gas-phase molecule $\mathbf{1 , 1}$ '-Fe for the angle $\varphi \simeq 150^{\circ}$ at which the distance between the SMe groups is minimal. The HOMO-3 orbital shows substantial overlap between the phenyl arms of the molecule and between the SMe groups. 
(a)
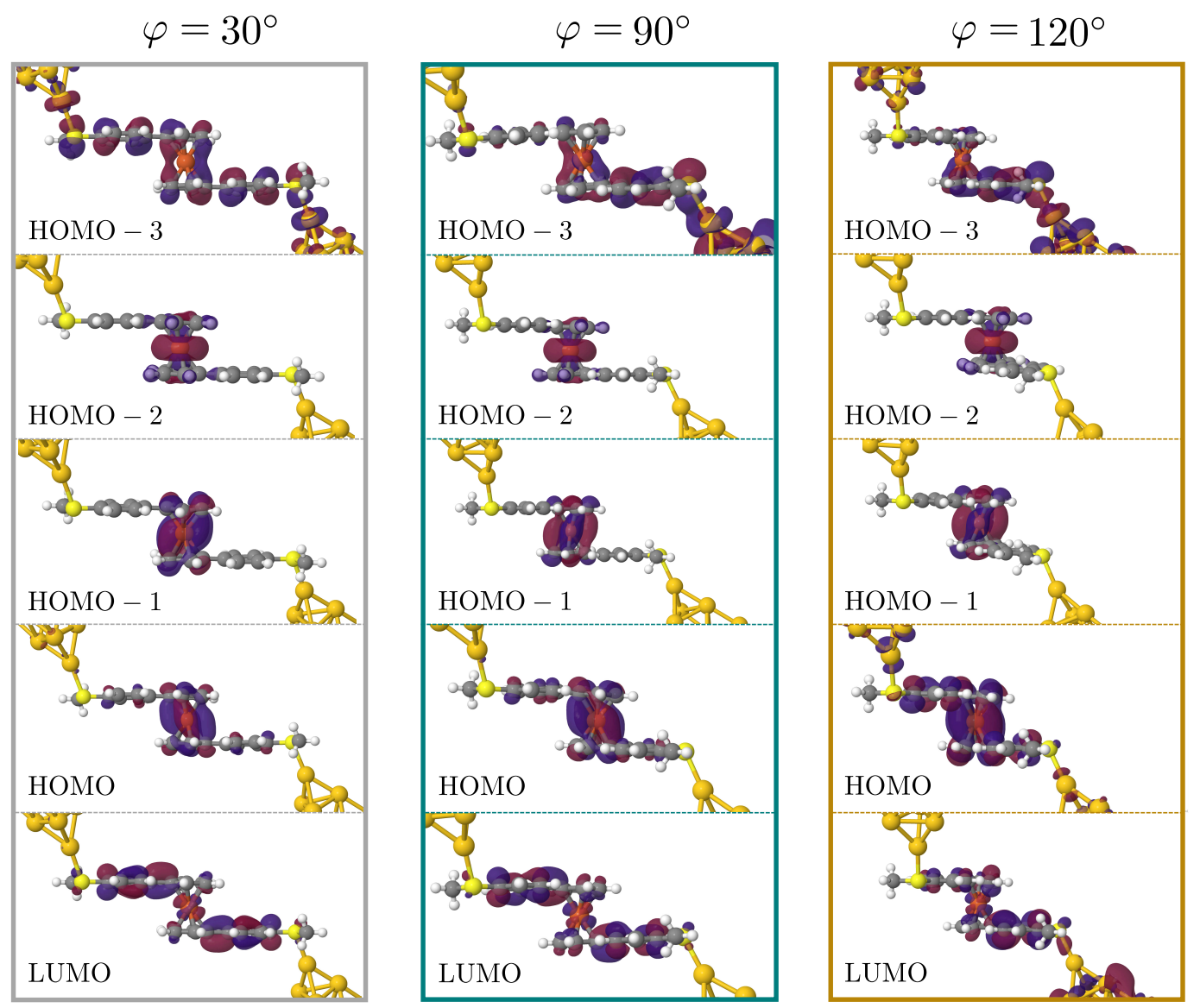

(b)
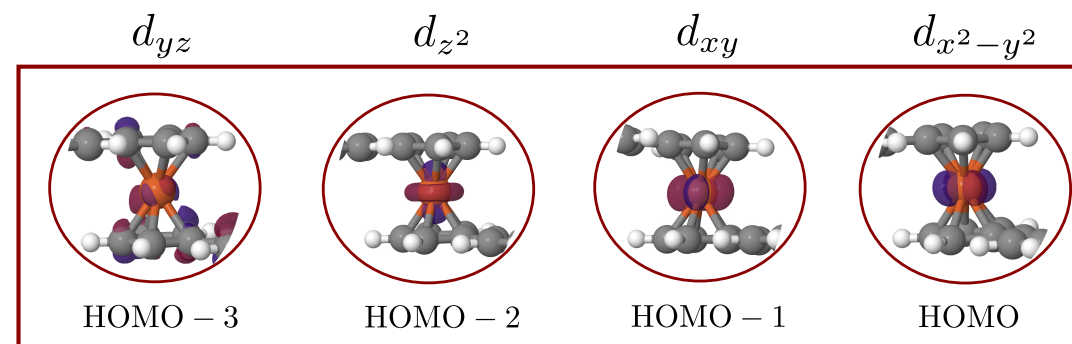

$\mathrm{HOMO}$

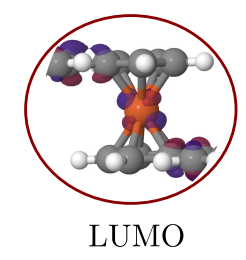

Fig. S6. (a) Selection of isosurface plots of the Kohn-Sham orbitals for the 1,1'-Fe junction close to the Fermi energy. (b) Zoomed-in view of the scattering states for all orbitals shown in panel (a) for $\varphi=90^{\circ}$. The isosurface exhibits the d-orbital symmetry. 


\section{NMR SPECTRA}

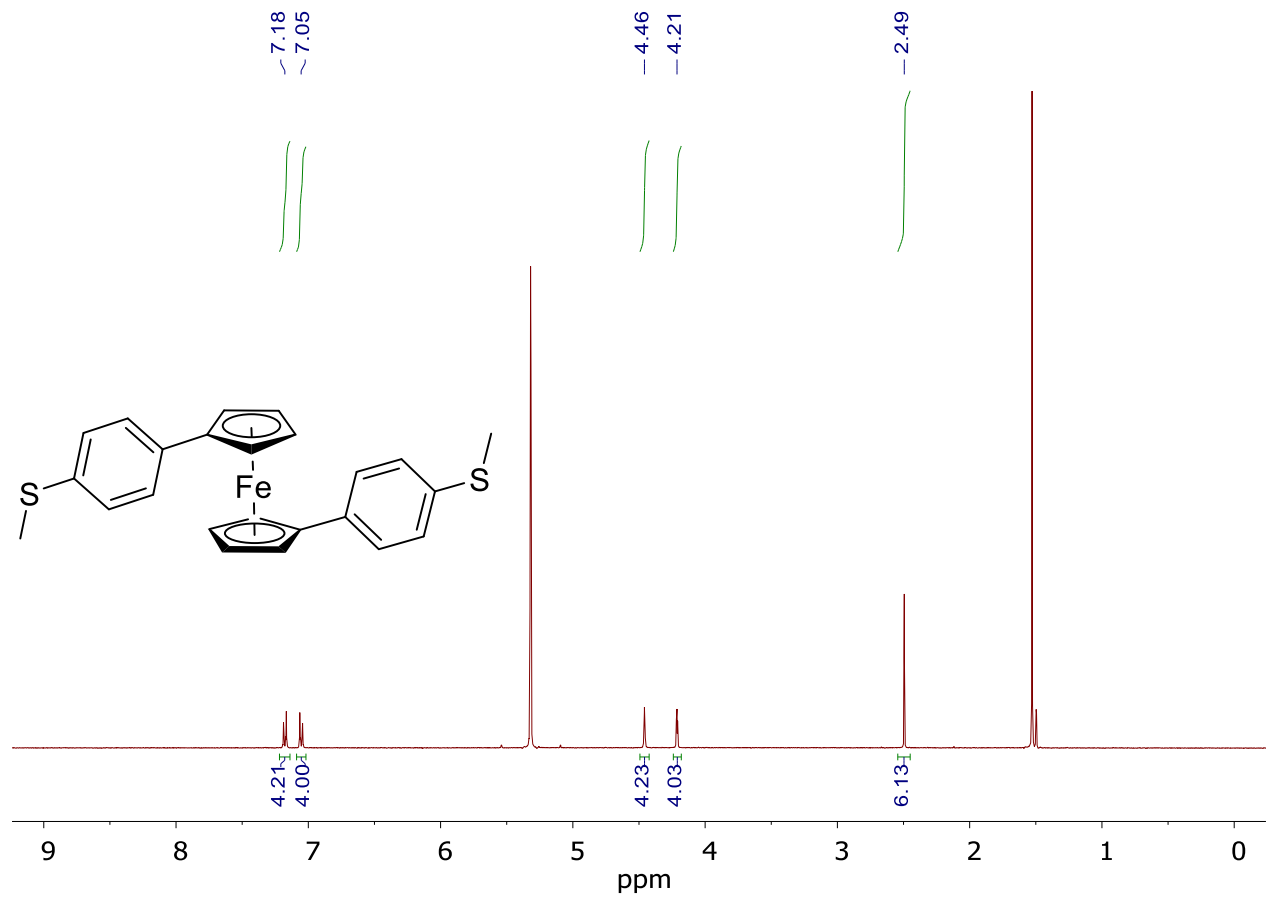

${ }^{1} \mathrm{H}$ NMR spectrum of $\mathbf{1 , 1 - F e}$ in $\mathrm{CD}_{2} \mathrm{Cl}_{2}$.

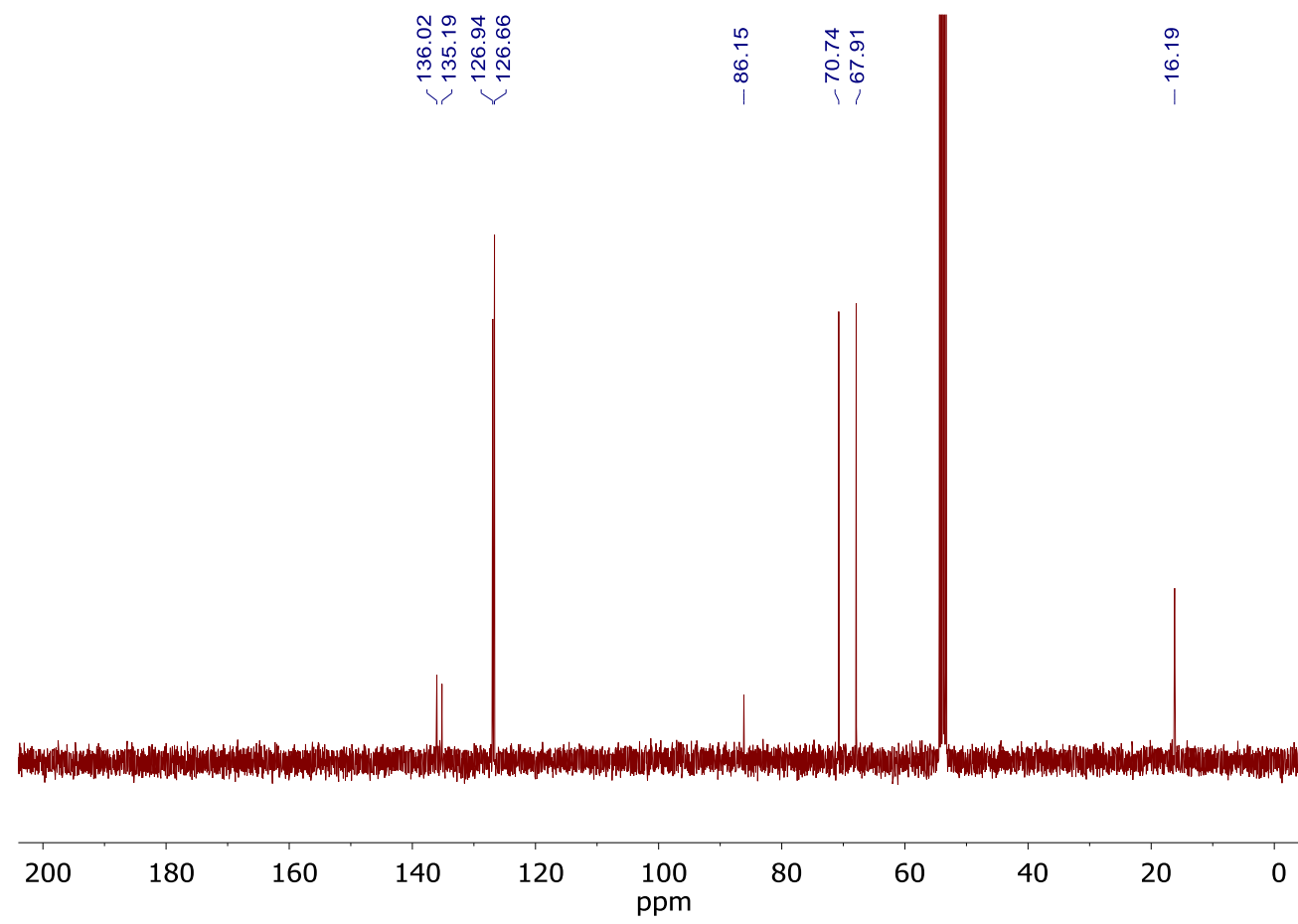

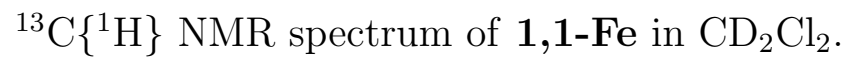




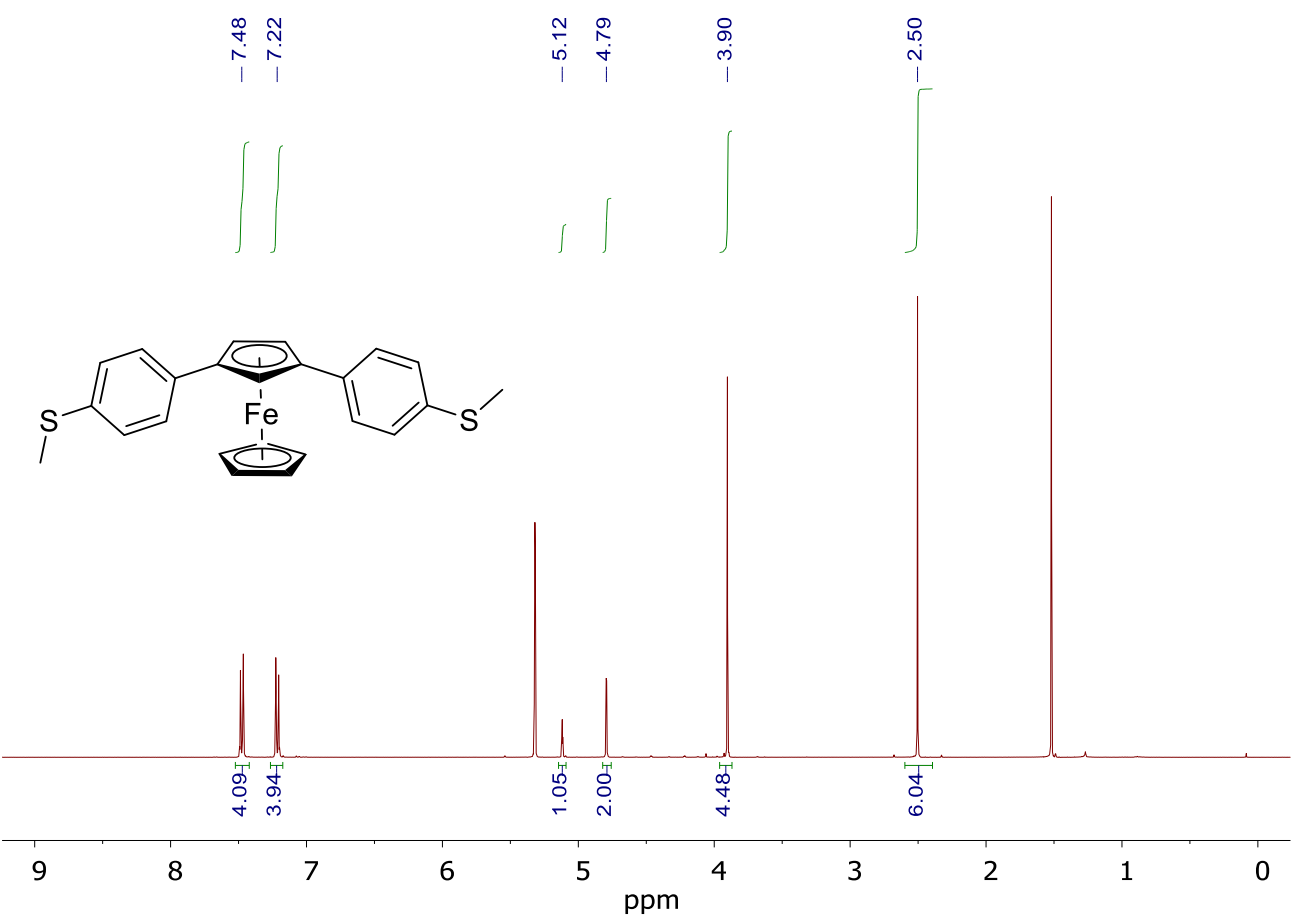

${ }^{1} \mathrm{H}$ NMR spectrum of $\mathbf{1 , 3 - F e}$ in $\mathrm{CD}_{2} \mathrm{Cl}_{2}$.

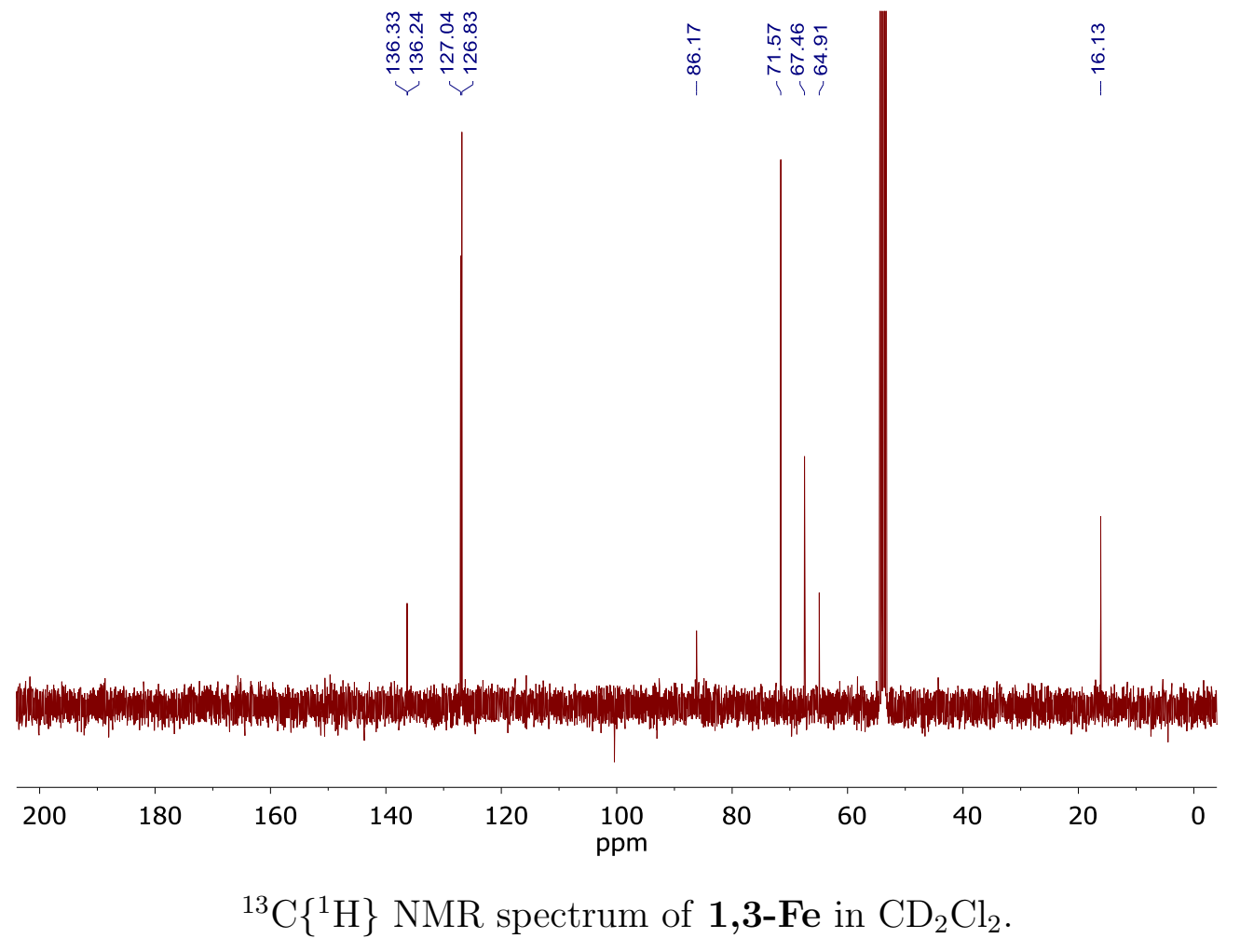


[1] A. Zirakzadeh, A. Herlein, M. Groß, K. Mereiter, Y. Wang, and W. Weissensteiner, Organometallics 34, 3820 (2015).

[2] H. V. Nguyen, M. Motevalli, and C. J. Richards, Synlett 2007, 725 (2007).

[3] S. P. H. Mee, V. Lee, and J. E. Baldwin, Ang. Chem. Int. Ed. 43, 1132 (2004).

[4] M. Enders, G. Kohl, and H. Pritzkow, Organometallics 21, 1111 (2002).

[5] H. K. Cotton, F. F. Huerta, and J.-E. Bäckvall, Eur. J. Org. Chem. 2003, 2756 (2003).

[6] G. K. Friestad, B. P. Branchaud, W. Navarrini, and M. Sansotera, "Cesium fluoride," in Encyclopedia of Reagents for Organic Synthesis (American Cancer Society, 2007).

[7] L. Venkataraman, J. E. Klare, I. W. Tam, C. Nuckolls, S. M. Hybertsen, and M. L. Steigerwald, Nano. Lett. 6, 458 (2006).

[8] V. Blum, R. Gehrke, F. Hanke, P. Havu, V. Havu, X. Ren, K. Reuter, and M. Scheffler, Comput. Phys. Comm. 180, 2175 (2009).

[9] J. P. Perdew, K. Burke, and M. Ernzerhof, Phys. Rev. Lett. 77, 3865 (1996).

[10] E. v. Lenthe, E. J. Baerends, and J. G. Snijders, J. Chem. Phys. 99, 4597 (1993).

[11] A. Arnold, F. Weigend, and F. Evers, J. Chem. Phys. 126, 174101 (2007).

[12] A. Bagrets, J. Chem. Theory Comput. 9, 2801 (2013). 
